\title{
Measurements of detector material samples with two HPGe detectors at the YangYang underground Laboratory
}

\section{Eunkyung Lee*, Eunju Jeon, Woon Gu Kang, Yeongduk Kim, Moo Hyun Lee, Douglas S. Leonard}

Center for Underground Physics, Institute for Basic Science (IBS),

Daejeon 34126, Republic of Korea

E-mail: freshblue78@gmail.com

\section{Kevin Insik Hahn}

Department of Science Education, Ewha Womans University,

Seoul 03760, Republic of Korea

\section{Gowoon Kim, Su-yeon Park}

Department of Physics, Ewha Womans University,

Seoul 03760, Republic of Korea

\section{Vladimir Kazalov}

Baksan Neutrino Observatory INR RAS,

Neutrino 361609, Russia

\begin{abstract}
Two major experiments, AMoRE searching for neutrino-less double beta decay and COSINE searching for dark matter WIMPs (Weakly Interacting Massive Particles), are running in the Yang Yang underground Laboratory (Y2L). To understand their signals, it is necessary to know the backgrounds from their detector materials such as fasteners, crystals, cables, connectors, etc. By using two 100\% HPGe detectors at the Y2L, the trace levels of naturally occurring radioactivity from the material samples were measured and analyzed using efficiencies estimated with the Geant4[1] simulation toolkit. In this report we present radioactivity measurements of the samples together with improved efficiency calibration produced with a mixed source containing 10 known radioactive isotopes.
\end{abstract}

The 39th International Conference on High Energy Physics (ICHEP2018)

4-11 July, 2018

Seoul, Korea

${ }^{*}$ Speaker. 


\section{Introduction}

The Center for Underground Physics (CUP) hosts two major rare-event search experiments, COSINE and AMoRE. Maximizing sensitivity to the signals of interest requires quantifying and reducing background signals arising from radioactive isotopes in the detector component materials such as mechanical and electrical parts, crystals, shielding materials, etc. These samples were measured with two 100\% HPGe detectors at Y2L, Canberra Coaxical detectors 1 and 2 (CC1, CC2). Efficiency discrepancies between simulations and HPGe measurements have previously been observed [2]. By tuning the Monte Carlo geometry to the data we produced efficiency estimates precise to within $10 \%$. As this was the dominant systematic uncertainly, activity measurements are generally precise to the same level.

\section{Experiment}

We used the Geant4.9.6 simulation toolkit to calculate efficiencies for various samples. Activities of samples were priviously calculated with $20 \%$ efficiency error before the efficiency calibration. As a source for the efficiency calibration, ten different isotopes with certified activities were mixed uniformly as a gel in a $1 \mathrm{~L}$ Marinelli beaker by KRISS. Mixed sources were measured with the $\mathrm{CC} 1$ and $\mathrm{CC} 2$ to calculate efficiencies of each detector. With the measured efficiencies, proper geometries of the $\mathrm{CC} 1$ and $\mathrm{CC} 2$ were estimated, reducing the Ge volumes $\left(\mathrm{CC} 1\right.$ : from $416.1 \mathrm{~cm}^{3}$ to $394.1 \mathrm{~cm}^{3}, \mathrm{CC} 2$ : from $408.3 \mathrm{~cm}^{3}$ to $383.8 \mathrm{~cm}^{3}$ ) by including inactive Ge layers (called dead layers). With updated efficiencies after adjusting the dead layers in the simulation code, activities of the samples were recalculated and compared with the previous analysis results. The resulting activities are slightly higher than previous estimates, but with reduced systematic uncertainties.

\section{Result}

\begin{tabular}{|c|c|c|c|c|cccc|}
\hline Detector & Sample \# & Sample name & Mass & Dead & \multicolumn{4}{|c|}{ Activity $(\mathrm{mBq} / \mathrm{kg})$} \\
\cline { 5 - 8 } & & & $(\mathrm{kg})$ & Layer & ${ }^{238} \mathrm{U}$ & ${ }^{40} \mathrm{~K}$ & ${ }^{228} \mathrm{Ac}$ & ${ }^{228} \mathrm{Th}$ \\
\hline $\mathrm{CC} 1$ & $\mathrm{CMD}$ & Refractory product & 0.223 & no DL & $385(38)$ & $689(157)$ & $565(86)$ & $480(50)$ \\
& 151.1 & (NaI grower) & & with DL & $496(30)$ & $791(95)$ & $676(50)$ & $555(33)$ \\
\hline $\mathrm{CC} 2$ & $\mathrm{CMD}$ & High Purity Alumina & 0.164 & no DL & $5.9(1.5)$ & $43.5(13.6))$ & $<5.0$ & $<4.4$ \\
& 162 & Refractory & & with DL & $7.0(1.2)$ & $51.0(12.6)$ & $<5.7$ & $<5.0$ \\
\hline
\end{tabular}

Table 1: Table of selected samples activities with and without the dead layers.

\section{References}

[1] S. Agostinelli et al., Geant4 - a simulation toolkit, NIM A 506 (2003) 250

[2] N. Q. Huy et al., Study on the increase of inactive germanium layer in a high-purity germanium detector after a long time operation applying MCNP code, NIM A 753 (2007) 384

[3] E. Sala et al., Development of an underground low background instrument for high sensitivity measurements, J. Phys.: Conf. Ser. 718 (2016) 062050. 